\title{
ATTACHED PRIMES OF THE TOP GENERALIZED LOCAL COHOMOLOGY MODULES
}

\author{
YAN GU ${ }^{凶}$ and LIZHONG CHU
}

\author{
(Received 2 April 2008)
}

\begin{abstract}
Let $(R, \mathfrak{m})$ be a commutative Noetherian local ring, let $I$ be an ideal of $R$ and let $M$ and $N$ be finitely generated $R$-modules. Assume that $\operatorname{pd}(M)=d<\infty, \operatorname{dim} N=n<\infty$. First, we give the formula for the attached primes of the top generalized local cohomology module $H_{I}^{d+n}(M, N)$; later, we prove that if $\operatorname{Att}\left(H_{I}^{d+n}(M, N)\right)=\operatorname{Att}\left(H_{J}^{d+n}(M, N)\right)$, then $H_{I}^{d+n}(M, N)=H_{J}^{d+n}(M, N)$.

2000 Mathematics subject classification: primary 13D45; secondary 13D07.

Keywords and phrases: generalized local cohomology modules, attached primes.
\end{abstract}

\section{Introduction}

Throughout this paper, let $(R, \mathfrak{m})$ be a commutative Noetherian local ring, let $I$ be a proper ideal of $R$ and let $M$ and $N$ be finitely generated $R$-modules. The generalized local cohomology module

$$
H_{I}^{i}(M, N)=\underset{n \in \mathbb{N}}{\lim } \operatorname{Ext}_{R}^{i}\left(M / I^{n} M, N\right)
$$

was introduced by Herzog [10] and studied further by Yassemi, Suzuki and so on. There are several well-known properties concerning the generalized local cohomology modules. Assume that $\operatorname{pd}(M)=d<\infty, \operatorname{dim} N=n<\infty$. It is well known that $H_{I}^{i}(M, N)=0$, for all $i>d+n$.

Recall that for an $R$-module $K$, a prime ideal $p$ of $R$ is said to be an attached prime of $K$, if $p=\operatorname{Ann}(K / L)$ for some submodule $L$ of $K$ (see [11]). The set of attached primes of $K$ is denoted by $\operatorname{Att}(K)$. If $K$ is an Artinian $R$-module, then $K$ admits a reduced secondary representation $K=K_{1}+\cdots+K_{r}$ such that $K_{i}$ is $p_{i}$-secondary, $i=1, \ldots, r$, then $\operatorname{Att}(K)=\left\{p_{1}, \ldots, p_{r}\right\}$ is a finite set. Note that $\operatorname{Att}(K)=\emptyset$ if and only if $K=0$. If $\operatorname{dim} M=n<\infty$, it is well known that $H_{I}^{n}(M)$ is an Artinian module. In [11], Macdonald and Sharp studied $H_{\mathfrak{m}}^{n}(M)$ and proved that

$$
\operatorname{Att}\left(H_{\mathfrak{m}}^{n}(M)\right)=\{p \in \operatorname{Ass}(M) \mid \operatorname{dim} R / p=n\}
$$

(C) 2009 Australian Mathematical Society 0004-9727/2009 \$16.00 
(the right-hand set is denoted by $\operatorname{Assh}(M))$. Dibaei and Yassemi [6, Theorem A] generalized this result to $\operatorname{Att}\left(H_{I}^{n}(M)\right)=\{p \in \operatorname{Ass}(M) \mid \operatorname{cd}(I, R / p)=n\}$, where for any $R$-module $K, \operatorname{cd}(I, K)=\sup \left\{i \in \mathbb{Z} \mid H_{I}^{i}(K) \neq 0\right\}$.

The object of this paper is the attached primes of the top generalized local cohomology modules $H_{I}^{d+n}(M, N)$, and we give a formula for $\operatorname{Att}\left(H_{I}^{d+n}(M, N)\right)$, which generalizes $[6$, Theorem A].

Let $E=E_{R}(R / \mathfrak{m})$, the injective hull of $R / \mathfrak{m}$. As in [14], we define a prime $p$ to be a coassociated prime of $L$ if $p$ is an associated prime of $D(L)$, where $D(\cdot)$ is Matlis' dual functor $\operatorname{Hom}(\cdot, E)$.

It is well known that for any integer $i$, there is an exact sequence

$$
H_{\mathfrak{m}}^{i}(M) \rightarrow H_{I}^{i}(M) \rightarrow \underset{t \in \mathbb{N}}{\lim } \operatorname{Ext}_{R}^{i}\left(\mathfrak{m}^{t} / I^{t}, M\right) .
$$

By Hartshorne's result (see [8]), we know that the right-hand side module in (1) is zero, so there exists an exact sequence $H_{\mathfrak{m}}^{n}(M) \rightarrow H_{I}^{n}(M) \rightarrow 0$. So one can deduce that for two ideals $I \subseteq J$, there exists an exact sequence $H_{J}^{n}(M) \rightarrow H_{I}^{n}(M) \rightarrow 0$.

For any pair ideals $I$ and $J$, if $\operatorname{Att}\left(H_{I}^{d+n}(M, N)\right)=\operatorname{Att}\left(H_{J}^{d+n}(M, N)\right)$, then we prove that $H_{I}^{d+n}(M, N)=H_{J}^{d+n}(M, N)$, from which we can obtain the result of [7].

\section{The formula for top generalized local cohomology modules}

In this section, we give the formula for the attached primes of the top generalized local cohomology module $H_{I}^{d+n}(M, N)$, and when $(R, \mathfrak{m})$ is a complete ring with respect to $\mathfrak{m}$-adic topology, we give the formula for $\operatorname{Coass}\left(H_{I}^{d+n}(M, N)\right)$.

The following lemma generalizes [13, Lemma 3.4].

LEMMA 2.1 [12, Lemma 2.8]. Let $\operatorname{pd}(M)=d<\infty$, L be an $R$-module and assume that $n \in \mathbb{N}$ and $x_{1}, \ldots, x_{n}$ is an I-filter regular sequence on L. Then

$$
H_{I}^{i+n}(M, L) \cong H_{I}^{i}\left(M, H_{\left(x_{1}, \ldots, x_{n}\right)}^{n}(L)\right), \quad \forall i \geq d .
$$

The next result is important for the main results of this paper.

Proposition 2.2. Assume that $\operatorname{pd}(M)=d<\infty, \operatorname{dim} N=n<\infty$. Then:

(i) $H_{I}^{d+n}(M, N) \cong \operatorname{Ext}_{R}^{d}\left(M, H_{I}^{n}(N)\right)$; in particular, $H_{I}^{d+n}(M, N)$ is Artinian and I-cofinite;

(ii) $\operatorname{Att}\left(H_{I}^{d+n}(M, N)\right) \subseteq \operatorname{Att}\left(H_{I}^{n}(N)\right)$.

PROOF. (i) Let $x_{1}, \ldots, x_{n}$ be an $I$-filter regular sequence on $N$. Then

$$
H_{I}^{d+n}(M, N) \cong H_{I}^{d}\left(M, H_{\left(x_{1}, \ldots, x_{n}\right)}^{n}(N)\right)
$$

by Lemma 2.1. By [4, Exercise 7.1.7], $H_{\left(x_{1}, \ldots, x_{n}\right)}^{n}(N)$ is Artinian. So by [13, Lemma 3.4] $H_{\left(x_{1}, \ldots, x_{n}\right)}^{n}(N) \cong H_{I}^{0}\left(H_{\left(x_{1}, \ldots, x_{n}\right)}^{n}(N)\right) \cong H_{I}^{n}(N)$. Therefore,

$$
H_{I}^{d+n}(M, N) \cong H_{I}^{d}\left(M, H_{\left(x_{1}, \ldots, x_{n}\right)}^{n}(N)\right) \cong H_{I}^{d}\left(M, H_{I}^{n}(N)\right) \cong \operatorname{Ext}_{R}^{d}\left(M, H_{I}^{n}(N)\right) .
$$


(ii) Suppose that $p \in \operatorname{Att}\left(H_{I}^{d+n}(M, N)\right)$, then

$$
H_{I}^{d+n}(M, N) / p H_{I}^{d+n}(M, N) \neq 0 .
$$

By (i), we have that

$$
H_{I}^{d+n}(M, N) / p H_{I}^{d+n}(M, N) \cong \operatorname{Ext}_{R}^{d}\left(M, H_{I}^{n}(N)\right) \otimes(R / p) .
$$

Since $\operatorname{Ext}_{R}^{d}(M,-)$ is a right exact additive functor,

$$
\begin{aligned}
\operatorname{Ext}_{R}^{d}\left(M, H_{I}^{n}(N)\right) \otimes(R / p) & \cong \operatorname{Ext}_{R}^{d}(M, R) \otimes H_{I}^{n}(N) \otimes(R / p) \\
& \cong \operatorname{Ext}_{R}^{d}\left(M, H_{I}^{n}(N) / p H_{I}^{n}(N)\right),
\end{aligned}
$$

thus $H_{I}^{n}(N) / p H_{I}^{n}(N) \neq 0$, hence $p \in \operatorname{Att}\left(H_{I}^{n}(N)\right)$.

Theorem 2.3. Assume that $\operatorname{pd}(M)=d<\infty$, $\operatorname{dim} N=n<\infty$. Then

$$
\operatorname{Att}\left(H_{I}^{d+n}(M, N)\right)=\{p \in \operatorname{Ass}(N) \mid \operatorname{cd}(I, M, R / p)=d+n\}
$$

where, for any $R$-module $K$,

$$
\operatorname{cd}(I, M, K)=\sup \left\{i \in \mathbb{Z} \mid H_{I}^{i}(M, K) \neq 0\right\} .
$$

ProOF. We use induction on $n$. If $n=0$, then $\lambda(N)<\infty$. So

$$
\operatorname{Att}\left(H_{I}^{0}(N)\right)=\operatorname{Att}(N)=\{\mathfrak{m}\}=\operatorname{Ass}(N)=\operatorname{Supp}(N),
$$

thus, $\operatorname{Att}\left(H_{I}^{d}(M, N)\right) \subseteq \operatorname{Att}\left(H_{I}^{0}(N)\right)=\{\mathfrak{m}\}=\operatorname{Ass}(N) \quad$ (where the containment follows from Proposition 2.2(ii)).

(1) If $H_{I}^{d}(M, N)=0$, then $\operatorname{Att}\left(H_{I}^{d}(M, N)\right)=\emptyset, \quad \operatorname{cd}(I, M, N)<d$, thus $\operatorname{cd}(I, M, R / \mathfrak{m})<d$ by [1, Proposition 2].

(2) If $H_{I}^{d}(M, N) \neq 0$, then $\operatorname{Att}\left(H_{I}^{d}(M, N)\right)=\{\mathfrak{m}\}=\operatorname{Ass}(N)$ and $\operatorname{cd}(I, M, N)=$ $d$, thus $\operatorname{cd}(I, M, R / \mathfrak{m})=d$ by [1, Proposition 2]. So the result has been proved in this case.

Now let $n>1$ and the case $n-1$ is settled. If $H_{I}^{d+n}(M, N)=0$, then $\operatorname{cd}(I, M, N)<d+n$, so $\{p \in \operatorname{Ass}(N) \mid \operatorname{cd}(I, M, R / p)=d+n\}=\emptyset$ by $[1$, Theorem B]. Now let $H_{I}^{d+n}(M, N) \neq 0$, let $L$ be the largest submodule of $N$ with $\operatorname{cd}(I, M, L)<d+n$. By the short exact sequence $0 \rightarrow L \rightarrow N \rightarrow N / L \rightarrow 0$ and [1, Theorem A], we have $\operatorname{cd}(I, M, N)=\operatorname{cd}(I, M, N / L)$. It is easy to prove that $N / L$ has no nonzero submodule $K$ with $\operatorname{cd}(I, M, K)<\operatorname{cd}(I, M, N)$, so

$$
\operatorname{Ass}(N / L) \subseteq\{p \in \operatorname{Supp}(N / L) \mid \operatorname{cd}(I, M, R / p)=d+n\} .
$$

In addition, if $p \in \operatorname{Supp}(N / L)$ and $\operatorname{cd}(I, M, R / p)=d+n$, then

$$
\begin{aligned}
d+n=\operatorname{cd}(I, M, R / p) & \leq d+\operatorname{dim} R / p \\
& \leq d+\operatorname{dim} N / L \leq d+\operatorname{dim} N=d+n
\end{aligned}
$$


Therefore, $p \in \min (\operatorname{Supp}(N / L)) \subseteq \operatorname{Ass}(N / L)$, and $p \in \min (\operatorname{Supp}(N)) \subseteq \operatorname{Ass}(N)$, therefore

$$
\begin{aligned}
\operatorname{Ass}(N / L) & =\{p \in \operatorname{Supp}(N / L) \mid \operatorname{cd}(I, M, R / p)=d+n\} \\
& \subseteq\{p \in \operatorname{Ass}(N) \mid \operatorname{cd}(I, M, R / p)=d+n\}
\end{aligned}
$$

If $p \in \operatorname{Ass}(N)$, and $\operatorname{cd}(I, M, R / p)=d+n$, then $p \notin \operatorname{Supp}(L)$, otherwise, $\operatorname{cd}(I, M, R / p) \leq \operatorname{cd}(I, M, L)<d+n$ by [1, Theorem B]. So $p \in \operatorname{Supp}(N / L)$, hence

$$
\begin{aligned}
\{p & \in \operatorname{Supp}(N / L) \mid \operatorname{cd}(I, M, R / p)=d+n\} \\
& =\{p \in \operatorname{Ass}(N) \mid \operatorname{cd}(I, M, R / p)=d+n\} .
\end{aligned}
$$

In the following exact sequence

$$
H_{I}^{d+n}(M, L) \rightarrow H_{I}^{d+n}(M, N) \rightarrow H_{I}^{d+n}(M, N / L) \rightarrow H_{I}^{d+n+1}(M, L),
$$

since $H_{I}^{d+n}(M, L)=H_{I}^{d+n+1}(M, L)=0$, we have

$$
H_{I}^{d+n}(M, N) \cong H_{I}^{d+n}(M, N / L) .
$$

Since $n=\operatorname{dim} N / L$,

$$
\begin{aligned}
& \{p \\
& \quad=\{p \in \operatorname{Ass}(N / L) \mid \operatorname{cd}(I, M, R / p)=d+n\} \\
& \quad\{(N) \mid \operatorname{cd}(I, M, R / p)=d+n\},
\end{aligned}
$$

we can assume that $L=0$, that is, $N$ has no nonzero submodule $L$ such that $\operatorname{cd}(I, M, L)<d+n$. Next we prove that $\operatorname{Att}\left(H_{I}^{d+n}(M, N)\right)=\operatorname{Ass}(N)$.

By Proposition 2.2(ii), we have that $\operatorname{Att}\left(H_{I}^{d+n}(M, N)\right) \subseteq \operatorname{Att}\left(H_{I}^{n}(N)\right) \subseteq \operatorname{Ass}(N)$.

On the other hand, if $p \in \operatorname{Ass}(N)$, then there is a $p$-primary submodule $T$ of $N$ such that $\operatorname{Ass}(N / T)=\{p\}$. We have $\operatorname{cd}(I, M, N / T) \geq \operatorname{cd}(I, M, R / p)=d+n$ by $\left[1\right.$, Theorem B], then $\operatorname{cd}(I, M, N / T)=d+n, \quad H_{I}^{\bar{d}+n}(M, N / T) \neq 0 . \quad$ By Proposition 2.2(ii) we obtain

$$
\operatorname{Att}\left(H_{I}^{d+n}(M, N / T)\right) \subseteq \operatorname{Att}\left(H_{I}^{n}(N / T)\right) \subseteq \operatorname{Ass}(N / T)=\{p\},
$$

so $\{p\}=\operatorname{Att}\left(H_{I}^{d+n}(M, N / T)\right)$. Considering the exact sequence $H_{I}^{d+n}(M, N)$ $\rightarrow H_{I}^{d+n}(M, N / T) \rightarrow 0$, then we have $\{p\} \subseteq \operatorname{Att}\left(H_{I}^{d+n}(M, N)\right)$, hence $\operatorname{Ass}(N)$ $\subseteq \operatorname{Att}\left(H_{I}^{d+n}(M, N)\right)$. Now the proof is complete.

REMARK 2.4. Assuming $\operatorname{pd}(M)=d<\infty, \operatorname{dim} N=n<\infty$. If

$$
p \in \operatorname{Att}\left(H_{I}^{d+n}(M, N)\right),
$$

then

$$
d+n=\operatorname{cd}(I, M, R / p) \leq d+\operatorname{dim} R / p \leq d+\operatorname{dim} N=d+n .
$$

So $\operatorname{dim} R / p=n$, and hence $\operatorname{Att}\left(H_{I}^{d+n}(M, N)\right) \subseteq \operatorname{Assh}(N)$. 
In [6, Theorem B], Dibaei and Yassemi proved the following result. Let $L$ be a nonzero module (not necessarily finite) such that $\operatorname{dim} R=\operatorname{dim} L=n<\infty$. Then $\operatorname{Att}\left(H_{I}^{n}(L)\right) \subseteq\{p \in \operatorname{Ass}(L) \mid \operatorname{cd}(I, R / p)=n\}$.

Assuming that $L$ is finitely generated, we can obtain [6, Theorem A] by Theorem 2.3.

Corollary 2.5 [6, Theorem A]. Assume that $L$ is finitely generated, $\operatorname{dim} L=n$. Then $\operatorname{Att}\left(H_{I}^{n}(L)\right)=\{p \in \operatorname{Ass}(L) \mid \operatorname{cd}(I, R / p)=n\}$.

We know that for a ring $R$ with $\operatorname{dim} R>0$, if $H_{I}^{\operatorname{dim} R}(R) \neq 0$, then it is not finitely generated (see [4, Exercise 8.2.6]). As an application of Theorem 2.3, we have the following proposition.

Proposition 2.6. Assume that $\operatorname{pd}(M)=d<\infty, 0<\operatorname{dim} N=n<\infty$. If

$$
H_{\mathfrak{m}}^{d+n}(M, N) \neq 0,
$$

then it is not finitely generated.

Proof. As $H_{\mathfrak{m}}^{d+n}(M, N) \neq 0$, so $\operatorname{Att}\left(H_{\mathfrak{m}}^{d+n}(M, N)\right) \neq \emptyset$. We have

$$
\operatorname{Att}\left(H_{\mathfrak{m}}^{d+n}(M, N)\right) \subseteq \operatorname{Att}\left(H_{\mathfrak{m}}^{n}(N)\right) \subseteq\{p \in \operatorname{Ass}(N) \mid \operatorname{dim} R / p=n\}
$$

by Proposition 2.2(ii). Since $n>0$, then $\operatorname{Att}\left(H_{\mathfrak{m}}^{d+n}(M, N)\right) \nsubseteq\{\mathfrak{m}\}$. Since $H_{\mathfrak{m}}^{d+n}(M, N)$ is Artinian, it follows that $H_{\mathfrak{m}}^{d+n}(M, N)$ is not finitely generated by [4, Corollary 7.2.12].

In [5, Lemma 3], Delfino and Marley showed that, if $(R, \mathfrak{m})$ is a complete Noetherian local ring, $I$ an ideal of $R, M$ a finitely generated $R$-module of dimension $d$, then $\operatorname{Coass}\left(H_{I}^{d}(M)\right)=\{p \in V(\operatorname{Ann}(M)) \mid \operatorname{dim} R / p=d, \sqrt{I+p}=\mathfrak{m}\}$.

PROPOSITION 2.7. Let $(R, \mathfrak{m})$ be a complete ring with respect to the $\mathfrak{m}$-adic topology, assume that $\operatorname{pd}(M)=d<\infty, \operatorname{dim} N=n<\infty$. Then

$$
\operatorname{Coass}\left(H_{I}^{d+n}(M, N)\right) \subseteq\{p \in V(\operatorname{Ann}(N)) \mid \operatorname{dim} R / p=n, \sqrt{I+p}=\mathfrak{m}\} .
$$

Proof. Since $H_{I}^{d+n}(M, N)$ is Artinian, then

$$
\operatorname{Att}\left(H_{I}^{d+n}(M, N)\right)=\operatorname{Ass}\left(D\left(H_{I}^{d+n}(M, N)\right)=\operatorname{Coass}\left(H_{I}^{d+n}(M, N)\right) .\right.
$$

In addition, when $(R, \mathfrak{m})$ is a complete ring with respect to the $\mathfrak{m}$-adic topology, we can prove that if $p \in \operatorname{Ass}(N)$ with $\operatorname{cd}(I, M, R / p)=d+n$, then $p \in V(\operatorname{Ann}(N))$ with $\operatorname{dim} R / p=n$, and $\sqrt{I+p}=\mathfrak{m}$ by [4, Theorem 8.2.1]. So

$$
\operatorname{Coass}\left(H_{I}^{d+n}(M, N)\right) \subseteq\{p \in V(\operatorname{Ann}(N)) \mid \operatorname{dim} R / p=n, \sqrt{I+p}=\mathfrak{m}\}
$$

by Theorem 2.3. 
Proposition 2.8. Assume that $\operatorname{pd}(M)=d<\infty, \quad 1<\operatorname{dim} N=n<\infty$. Then $H_{I}^{d+n-1}(M, N) / I^{i} H_{I}^{d+n-1}(M, N)$ of finite length for any $i \in \mathbb{N}$.

Proof. We have that $H_{I}^{d+n}(M, N) \cong H_{I^{i}}^{d+n}(M, N)$ for all $i \in \mathbb{N}$, so it is enough to prove the result for $i=1$. By Proposition 2.2(i), $H_{I}^{d+n}(M, N)$ is Artinian and $I$-cofinite. In addition, we know that $H_{I}^{i}(M, N)=0$ for all $i>d+n$. By the same proof as in [2, Theorem 3.3], we get that $H_{I}^{d+n-1}(M, N) / I H_{I}^{d+n-1}(M, N)$ is Artinian and $I$-cofinite, so

$H_{I}^{d+n-1}(M, N) / I H_{I}^{d+n-1}(M, N) \cong \operatorname{Hom}\left(R / I, H_{I}^{d+n-1}(M, N) / I H_{I}^{d+n-1}(M, N)\right)$

is finitely generated, thus $H_{I}^{d+n-1}(M, N) / I H_{I}^{d+n-1}(M, N)$ has finite length.

ExAmple 2.9 [2, Example 3.6]. In Proposition 2.8, if $t<\operatorname{pd}(M)+\operatorname{dim} N-1$, then it can be seen that $H_{I}^{t}(M, N) / I H_{I}^{t}(M, N)$ is not necessarily of finite length. To see this, let $R=k\left[\left[X_{1}, \ldots, X_{4}\right]\right], I_{1}=\left(X_{1}, X_{2}\right), I_{2}=\left(X_{3}, X_{4}\right)$ and $I=I_{1} \cap I_{2}$, $M=N=R$, where $k$ is a field. Then $H_{I}^{i}(M, N)=H_{I}^{i}(R)$ for all $i \geq 0$. By the Mayer-Vietoris exact sequence we obtain that $H_{I}^{2}(R)=H_{I_{1}}^{2}(R) \oplus H_{I_{2}}^{2}(R)$. Now consider the following isomorphisms

$$
\begin{aligned}
H_{I}^{2}(R) / I H_{I}^{2}(R) & \cong\left(H_{I_{1}}^{2}(R) / I H_{I_{1}}^{2}(R)\right) \oplus\left(H_{I_{2}}^{2}(R) / I H_{I_{2}}^{2}(R)\right) \\
& =H_{I_{1}}^{2}(R / I) \oplus H_{I_{2}}^{2}(R / I)
\end{aligned}
$$

By the Hartshorne-Lichtenbaum vanishing theorem, $H_{I_{1}}^{2}(R / I) \neq 0$. Therefore, $\operatorname{cd}\left(I_{1}, R / I\right)=2$, and so by [9, Remark 2.5], $H_{I_{1}}^{2}(R / I)$ is not finitely generated. Consequently, $H_{I}^{2}(R) / I H_{I}^{2}(R)$ is not finitely generated.

\section{Top generalized local cohomology modules}

In [7, Theorem 1.6], Dibaei and Yssemi show that for any pair of ideals $I$ and $J, \operatorname{dim} N=n$, if $\operatorname{Att}\left(H_{I}^{n}(N)\right)=\operatorname{Att}\left(H_{J}^{n}(N)\right)$, then $H_{I}^{n}(N)=H_{J}^{n}(N)$. In this section, we show that, if $\operatorname{Att}\left(H_{I}^{d+n}(M, N)\right)=\operatorname{Att}\left(H_{J}^{d+n}(M, N)\right)$, then $H_{I}^{d+n}(M, N)$ $=H_{J}^{d+n}(M, N)$.

LemMa 3.1. Assume that $\operatorname{pd}(M)=d<\infty, \quad \operatorname{dim} N=n<\infty, \quad H_{I}^{d+n}(M, N) \neq 0$.

Then there exists a homomorphic image $G$ of $N$ such that:

(1) $\operatorname{dim} G=n$;

(2) $G$ has no nonzero submodule of dimension less than $n$;

(3) $\operatorname{Ass}(G)=\{p \in \operatorname{Ass}(N) \mid \operatorname{cd}(I, M, R / p)=d+n\}$;

(4) $H_{I}^{d+n}(M, G) \cong H_{I}^{d+n}(M, N)$;

(5) $\operatorname{Ass}(G)=\operatorname{Att}\left(H_{I}^{d+n}(M, G)\right)$. 
ProOF. By Remark 2.4, $\operatorname{Att}\left(H_{I}^{d+n}(M, N)\right) \subseteq \operatorname{Assh}(N)$. Therefore, there is a submodule $L$ of $N$ such that $\operatorname{Ass}(L)=\operatorname{Ass}(N) \backslash \operatorname{Att}\left(H_{I}^{d+n}(M, N)\right)$ and $\operatorname{Ass}(N / L)=\operatorname{Att}\left(H_{I}^{d+n}(M, N)\right)$ by [3, p. 263, Proposition 4]. Considering the exact sequence $H_{I}^{d+n}(M, L) \rightarrow H_{I}^{d+n}(M, N) \rightarrow H_{I}^{d+n}(M, N / L) \rightarrow 0$, we claim that $H_{I}^{d+n}(M, L)=0$. Otherwise, there is $p \in \operatorname{Att}\left(H_{I}^{d+n}(M, L)\right)$ such that $\operatorname{cd}(I, M, R / p)=d+n$. Since $p \in \operatorname{Ass}(L)$ by Theorem 2.3, then $p \in \operatorname{Ass}(N)$, and hence $p \in \operatorname{Att}\left(H_{I}^{d+n}(M, N)\right)$ by Theorem 2.3, which is a contradiction. Thus, $H_{I}^{d+n}(M, N)=H_{I}^{d+n}(M, N / L)$. Set $G=N / L$. Then (1), (3), (4), (5) are clear. If $G$ has a nonzero submodule $K$ with $\operatorname{dim} K<n$, then $\operatorname{dim} R / p<n$ for some $p \in \operatorname{Ass}(N / L)$, which is contradiction by Remark 2.4.

Proposition 3.2. Assume that $(R, \mathfrak{m})$ is a complete Noetherian local ring, $\operatorname{pd}(M)=d<\infty, \operatorname{dim} N=n<\infty$. Then $\operatorname{Att}\left(H_{I}^{d+n}(M, N)\right) \subseteq \operatorname{Att}\left(H_{J}^{d+n}(M, N)\right)$ if and only if $H_{J}^{d+n}(M, N) \rightarrow H_{I}^{d+n}(M, N) \rightarrow 0$ is an exact sequence.

ProOF. The sufficient part is clear. For the necessary part, there exists a submodule $L$ of $N$ with $\operatorname{Ass}(L)=\operatorname{Ass}(N) \backslash \operatorname{Att}\left(H_{J}^{d+n}(M, N)\right)$ and $\operatorname{Ass}(N / L)=$ $\operatorname{Att}\left(H_{J}^{d+n}(M, N)\right)$ by [3, p. 263, Proposition 4]. We see that $H_{J}^{d+n}(M, L)=0$ by the proof of Lemma 3.1. Hence, we have $H_{J}^{d+n}(M, N)=H_{J}^{d+n}(M, N / L)$. Note that for any $p \in \operatorname{Ass}(N / L)$ with $\operatorname{cd}(J, M, R / p)=d+n$, then $p \in \operatorname{Ass}(N), H_{J}^{n}(R / p) \neq 0$, so $J+p$ is $\mathfrak{m}$-primary by [4, Theorem 8.2.1]. This induces $J+\operatorname{Ann}(N / L)$ is $\mathfrak{m}$-primary and, hence,

$$
H_{J}^{d+n}(M, N / L) \cong H_{J+\operatorname{Ann}(N / L)}^{d+n}(M, N / L)=H_{\mathfrak{m}}^{d+n}(M, N / L) .
$$

On the other hand, considering the exact sequence

$$
H_{I}^{d+n}(M, L) \rightarrow H_{I}^{d+n}(M, N) \rightarrow H_{I}^{d+n}(M, N / L) \rightarrow 0,
$$

if $H_{I}^{d+n}(M, L) \neq 0$, then there exists $p \in \operatorname{Att}\left(H_{I}^{d+n}(M, L)\right)$. Then we have $p \in$ $\operatorname{Ass}(L)$ and $\operatorname{cd}(I, M, R / p)=d+n$ by Theorem 2.3. As $p \in \operatorname{Ass}(N)$, then $p \in$ $\operatorname{Att}\left(H_{I}^{d+n}(M, N)\right)$, thus $p \in \operatorname{Att}\left(H_{J}^{d+n}(M, N)\right)$, which contradicts with $p \in \operatorname{Ass}(L)$. Therefore, $H_{I}^{d+n}(M, L)=0$ and, hence, $H_{I}^{d+n}(M, N) \cong H_{I}^{d+n}(M, N / L)$. Since there exists an exact sequence

$$
H_{\mathfrak{m}}^{n}(N / L) \rightarrow H_{I}^{n}(N / L) \rightarrow 0, \quad H_{I}^{d+n}(M, N / L) \cong \operatorname{Ext}_{R}^{d}\left(M, H_{I}^{n}(N / L)\right)
$$

and

$$
H_{\mathfrak{m}}^{d+n}(M, N / L) \cong \operatorname{Ext}_{R}^{d}\left(M, H_{\mathfrak{m}}^{n}(N / L)\right)
$$

by Proposition 2.2(i), then $H_{J}^{d+n}(M, N) \rightarrow H_{I}^{d+n}(M, N) \rightarrow 0$ is an exact sequence. 
Theorem 3.3. Assume that $\operatorname{pd}(M)=d<\infty, \operatorname{dim} N=n<\infty$. If

$$
\operatorname{Att}\left(H_{I}^{d+n}(M, N)\right)=\operatorname{Att}\left(H_{J}^{d+n}(M, N)\right),
$$

then $H_{I}^{d+n}(M, N)=H_{J}^{d+n}(M, N)$.

Proof. Since $H_{I}^{d+n}(M, N)$ and $H_{J}^{d+n}(M, N)$ are Artinian, then

$$
H_{I \widehat{R}}^{d+n}(\widehat{M}, \widehat{N}) \cong H_{I}^{d+n}(M, N), \quad H_{J \widehat{R}}^{d+n}(\widehat{M}, \widehat{N}) \cong H_{J}^{d+n}(M, N),
$$

so we can assume that $R$ is complete. We take $L$ to be a submodule of $N$ such that $\operatorname{Ass}(N / L)=\operatorname{Att}\left(H_{I}^{d+n}(M, N)\right), \operatorname{Ass}(L)=\operatorname{Ass}(N) \backslash \operatorname{Att}\left(H_{I}^{d+n}(M, N)\right)$. By the following two exact sequences

$$
\begin{gathered}
H_{I}^{d+n}(M, L) \rightarrow H_{I}^{d+n}(M, N) \rightarrow H_{I}^{d+n}(M, N / L) \rightarrow 0, \\
H_{J}^{d+n}(M, L) \rightarrow H_{J}^{d+n}(M, N) \rightarrow H_{J}^{d+n}(M, N / L) \rightarrow 0 .
\end{gathered}
$$

As in the proof of Proposition 3.2, we obtain

$$
H_{I}^{d+n}(M, N)=H_{\mathfrak{m}}^{d+n}(M, N / L)=H_{J}^{d+n}(M, N) .
$$

In general, there exists an epimorphism $H_{\mathfrak{m}}^{n}(M) \rightarrow H_{I}^{n}(M)$, where $\operatorname{dim} M=n$. Next, in a particular case, we obtain that $H_{\mathfrak{m}}^{d+n}(M, N) \cong H_{I}^{d+n}(M, N)$, where $\operatorname{pd}(M)=d<\infty, \operatorname{dim} N=n<\infty$.

Proposition 3.4. Assume that $\operatorname{pd}(M)=d<\infty, \quad \operatorname{dim} N=n<\infty$ such that $\operatorname{Ass}(N)=\operatorname{Att}\left(H_{I}^{d+n}(M, N)\right)$. Then $H_{I}^{d+n}(M, N)=H_{\mathfrak{m}}^{d+n}(M, N)$.

PROOF. From

$$
\operatorname{Ass}(N)=\operatorname{Att}\left(H_{I}^{d+n}(M, N)\right) \subseteq \operatorname{Assh}(N) \subseteq \operatorname{Ass}(N),
$$

we have

$$
\operatorname{Att}\left(H_{I}^{d+n}(M, N)\right)=\operatorname{Assh}(N)=\operatorname{Att}\left(H_{\mathfrak{m}}^{n}(N)\right) \supseteq \operatorname{Att}\left(H_{\mathfrak{m}}^{d+n}(M, N)\right)
$$

by Theorem 2.3. On the other hand, since there exists an exact sequence $H_{\mathfrak{m}}^{n}(N) \rightarrow H_{I}^{n}(N) \rightarrow 0$, then $\operatorname{Ext}_{R}^{d}\left(M, H_{\mathfrak{m}}^{n}(N)\right) \rightarrow \operatorname{Ext}_{R}^{d}\left(M, H_{I}^{n}(N)\right) \rightarrow 0$ is an exact sequence, hence $H_{\mathfrak{m}}^{d+n}(M, N) \rightarrow H_{I}^{d+n}(M, N) \rightarrow 0$ is an exact sequence by Proposition 2.2(i). Therefore, $\operatorname{Att}\left(H_{\mathfrak{m}}^{d+n}(M, N)\right) \supseteq \operatorname{Att}\left(H_{I}^{d+n}(M, N)\right)$, so $H_{I}^{d+n}(M, N)=H_{\mathfrak{m}}^{d+n}(M, N)$ by Theorem 3.3.

\section{References}

[1] J. Amjadi and R. Naghipour, 'Cohomological dimension of generalized local cohomology modules', Algebra Colloq. (2) 15 (2008), 303-308.

[2] M. Asgharzadeh, K. Divaani-Aazar and M. Tousi, 'The finiteness dimension of local cohomology modules and its dual notion', J. Pure Appl. Algebra 213 (2009), 321-328. 
[3] N. Bourbaki, Commutative Algebra, Elements of Mathematics (Herman, Paris/Addison-Wesley, Reading, MA, 1972).

[4] M. P. Brodmann and R. Y. Sharp, Local Cohomology-An Algebraic Introduction with Geometric Applications, Cambridge Studies in Advanced Mathematics, 60 (Cambridge University Press, Cambridge, 1998).

[5] D. Delfino and T. Marley, 'Cofinite modules and local cohomology', J. Pure Appl. Algebra 121 (1997), 45-52.

[6] M. T. Dibaei and S. Yassemi, 'Attached primes of the top local cohomology modules with respect to an ideal', Arch. Math. 84 (2005), 292-297.

[7] _ 'Top local cohomology modules', Algebra Colloq. (2) 14(2) (2007), 209-214.

[8] R. Hartshorne, 'Cohomological dimension of algebraic varieties', Ann. of Math. 88 (1968), 403-450.

[9] M. Hellus, 'A note on the injective dimension of local cohomology modules', Proc. Amer. Math. Soc. 136 (2008), 2313-2321.

[10] J. Herzog, Komplex Auflösungen und Dualität in der lokalen Algebra (Habilitationsschrift, Universität Regensburg, 1974).

[11] I. G. Macdonald and R. Y. Sharp, 'An elementary proof of the non-vanishing of certain local cohomology modules', Quart. J. Math. Oxford 23 (1972), 197-204.

[12] A. Mafi, 'On the associated primes of generalized local cohomology modules', Comm. Algebra (7) 34 (2006), 2489-2494.

[13] U. Nagel and P. Schenzel, 'Cohomological annihilators and Castelnuovo Mumford regularity', in: Commutative Algebra: Syzygies, Multiplicities, and Birational Algebra (South Hadley, MA, 1992). Providence, RI, 1994, pp. 307-328.

[14] S. Yassemi, 'Coassociated primes', Comm. Algebra (4) 23 (1995), 1473-1498.

YAN GU, Department of Mathematics, Soochow University, 215006 Suzhou, Jiangsu, People's Republic of China

e-mail: guyan@suda.edu.cn

LIZHONG CHU, Department of Mathematics, Soochow University, 215006 Suzhou, Jiangsu, People's Republic of China

e-mail: chulizhong@suda.edu.cn 